\title{
Advances in the diagnosis and treatment of leprosy
}

\section{Vishwa M. Katoch}

Leprosy is a chronic infectious disease caused by Mycobacterium leprae that mainly affects the skin and peripheral nerves. Over recent years, many important advances have been made in developing molecular diagnostics, in identifying highly effective drugs and designing multidrug regimens for treatment, and in unravelling the genomic structure and functions of the leprosy bacillus. Using the new information about specific sequences of $M$. leprae, several gene probes and gene amplification systems for confirming diagnosis and monitoring treatment have been developed. Among these, polymerase chain reaction (PCR)based methods have been useful in confirming the diagnosis in paucibacillary leprosy (where few bacilli are present). RNA-targeting systems for monitoring the progress of treatment, in situ hybridisation techniques for analysing specimens with nonspecific histological features, and molecular methods for direct detection of rifampicin/dapsone resistance are other major technological advances with immense applied value. Several effective regimens for the treatment of leprosy have been developed, which include rifampicin, clofazimine and dapsone as core drugs. Although these regimens are generally satisfactory, limitations in terms of persisting activity and late reactions/relapses in paucibacillary leprosy, and persistence of dead and/or live organisms in multibacillary forms of the disease, have been observed.

The demonstration of the bacterial aetiology of leprosy in 1873 by the Norwegian Armauer Hansen (yielding the alternative names for leprosy of Hansen's disease and Hanseniasis) is considered one of the important landmarks in the arena of infectious diseases. However, because it has not been possible to cultivate Mycobacterium leprae in vitro, progress in understanding the biology of leprosy bacillus has been very slow. The success achieved in growing leprosy bacillus in the mouse foot (Ref. 1) galvanised leprosy research, leading to extensive work in different animal models, development of new chemotherapeutic agents, and the analysis of the biochemical, antigenic and molecular structure of leprosy bacillus. These advances have culminated in important developments in terms of both molecular diagnostics for early diagnosis and effective regimens for the treatment of leprosy. This article briefly reviews the aetiopathology of

Vishwa M. Katoch

Director, Central JALMA Institute for Leprosy (Indian Council of Medical Research), PO Box 101, Taj Ganj, Agra 282 001, India. Tel: +91 562 331381/331756; Fax: +91 562331 755; E-mail: jalma@zyberway.com 
leprosy before focusing on recent advances in the diagnosis and treatment of leprosy.

\section{M. leprae}

\section{Aetiopathology of leprosy: an introduction}

M. leprae, a member of the family Mycobacteriacae, is a straight or slightly curved rod-shaped organism, $1-8 \mu \mathrm{m}$ long and $0.3 \mu \mathrm{m}$ in diameter. It divides by binary fission, is Gram-positive, and strongly 'acid fast' following staining with basic fuchsin, which stains the bacteria pink. (In contrast, staining of $M$. tuberculosis is both acid fast and alcohol fast.) M. leprae is an obligate intracellular parasite, found predominantly in macrophages, where the organism commonly occurs in clumps or 'globi'. The optimal growth of $M$. leprae is observed at $27-30^{\circ} \mathrm{C}$, which is also reflected clinically, as the cooler areas of the body such as the skin, nasal mucosa, and superficial peripheral nerves (particularly Schwann cells) are the predominant sites of infection.

\section{Epidemiology}

The current geographical distribution of leprosy is shown in Figure 1. The global burden of the disease has decreased tremendously since the introduction of multidrug treatment (MDT) in 1982. Nearly half a million cases are estimated to remain worldwide, mainly in the Asian and African subcontinents (Table 1). Countries where leprosy continues to be a major problem include Brazil and India. Although no non-human sources of infection have been established, naturally occurring infection in monkeys and armadillos has been reported. $M$. leprae-like organisms have also been reported to be present in soil. The mode of transmission of leprosy has not been fully established, but nose and skin are considered as the main portals of exit as well as entry (Ref. 2).

\section{Clinical features and classification}

The incubation period of the disease is long and highly variable (usually 2-10 years). Most individuals exposed to infection are naturally

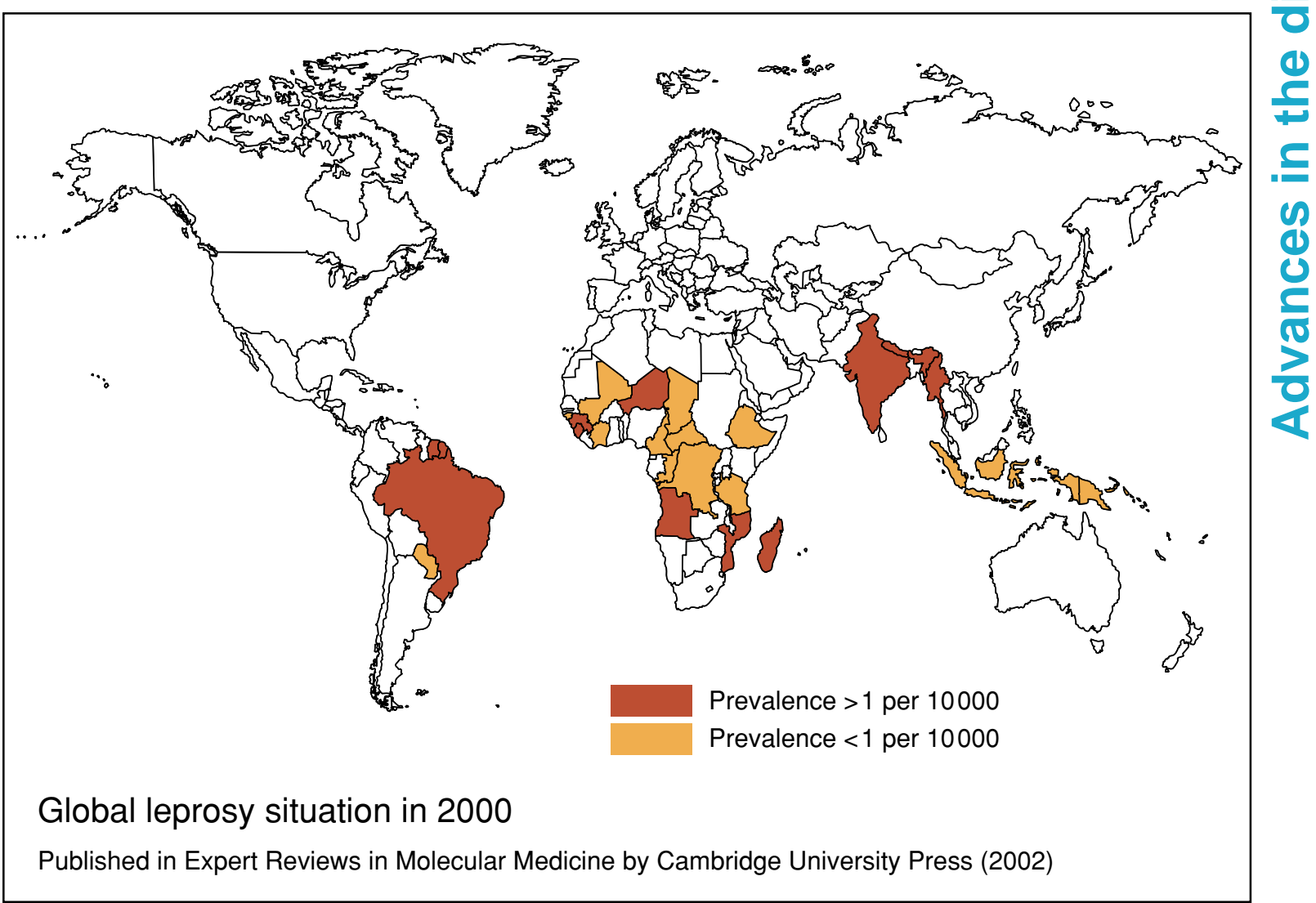

Figure 1. Global leprosy situation in 2000. Countries with a leprosy prevalence rate of more than 1 in 10000 include Brazil, India and central and southern African countries. Map reproduced, with permission, from http://www.who.int/lep/12.htm (Ref. 98) (fig001vka).

Accession information: (02)00476-3a.pdf (short code: txt001vka); 22 J uly 2002 
protected, are able to mount an efficient immune response, and do not suffer from the disease. In those who suffer from the disease, the main clinical features in paucibacillary (PB) disease (see below) result from damage due to immune responses mounted by the host, whereas in the lepromatous (LL) forms bacillary load and to some extent immune response are responsible for the clinical presentation. Symptoms and signs pertaining to involvement of the skin and nerves are most commonly encountered, including hypopigmented macules and sensory loss (Ref. 3). At least two of the following findings have to be present for a clinical diagnosis of leprosy: (1) a characteristic patch or skin lesion with impaired sensations; (2) a thickened and/or tender cutaneous or peripheral nerve with impairment of sensations in the area supplied by it; and (3) acid-fast bacteria in the skin smear.

The disease is formally classified into a range of subtypes that include, in approximate order of extent of disease, 'suspicious', early indeterminate (I), tuberculoid (TT), borderline tuberculoid (BT), mid borderline (BB), borderline lepromatous (BL) and LL disease (Ref. 4). Other forms, such as pure neurotic leprosy (without skin lesions), are also recognised (Ref. 5). The degree and type of immune response and also probably the route of infection determine the clinical forms of the disease. Individuals with strong cell-mediated immunity (CMI) or delayed-type hypersensitivity (DTH) show a localised TT disease, whereas individuals lacking CMI progress from I to LL disease. The immune defect is specific to M. leprae; other immune responses remain intact. The disease is characterised by the formation of granulomas; these vary from epithelioid type in TT leprosy to foamy cell (macrophage) type in LL disease (Ref. 6).

The lepromin skin test, which measures DTH to $M$. leprae antigens, is used to assist diagnosis and classification of leprosy (Ref. 7). The response is biphasic, with an early response at $24-48 \mathrm{~h}$, and a late response at 3-4 weeks. Bacterial load in the disease can be estimated from a smear, taken from skin lesions, stained for acid-fast bacteria. Density of bacteria (both viable and nonviable) is expressed using the logarithmic Ridley Scale [bacterial index (BI) 0-6] (Ref. 8). For treatment purposes, smear-positive BT, BB, BL and LL cases are referred to as multibacillary (MB) leprosy, whereas smear-negative I, TT and BT cases are termed PB leprosy (Ref. 9).

\section{Reactions and relapses}

Episodes of acute inflammation in the leprosy lesions and/or in nerves and other body parts have been popularly referred to as 'reactions'. It is hypothesised that these are brought about by disturbances in immunological balance as a result of immune reactivity to $M$. leprae antigens. Identical antigenic determinants of the host might also contribute to the autoimmune phenomenon. Three types of reactions are recognised. (1) Type I 
(reversal) reactions are associated with changes in CMI; BT, BB and BL patients usually suffer from these reactions, and if not promptly treated the reactions can result in nerve damage and deformities. (2) Type II reactions [erythema nodosum leprosum (ENL) reactions] affect patients with $\mathrm{MB}$ disease (BL and LL, and sometimes $\mathrm{BB}$ ) and are thought to arise from deposition of immune complexes in target organs and skin. (3) The Lucio phenomenon, which is less well understood, is associated with extensive skin necrosis due to acute vasculitis and occlusion of arterioles whose endothelium is massively invaded by $M$. leprae. In addition to these classifications, episodes of inflammation or reactions occurring during treatment are known as 'early reactions', whereas when the same occur after stoppage of treatment they are known as 'late reactions'. It is thought that late reactions are caused by dead bacteria or antigens.

Relapse in leprosy is defined as recrudescence of the disease activity after successful completion of a prescribed course of therapy. This is considered to result from multiplication of the few remaining live organisms. As there is an element of inflammation in the re-activation and appearance of new lesions, relapses are often confused with late reactions (presumed to be due to dead organisms). Clinically these two conditions overlap, and histology is also not always helpful in distinguishing the two. Currently available methods for determination of viability of $M$. leprae (including the mouse footpad assay, and bacillary ATP and substrate uptake assays) are reliable only in MB disease (Ref. 10).

\section{Molecular techniques for diagnosis and monitoring of treatment for leprosy}

Standard immunological and histological approaches for assessing leprosy have limited value for diagnosing new cases at the 'suspicious' and early I stages and for monitoring treatment. Immunological techniques for eliciting DTH and serological responses in leprosy are useful only for determining exposure, as the antigens and resultant response persist for a long time after subsidence of clinical or subclinical disease. Demonstration of acid-fast bacteria in skin smears is also often not sufficiently sensitive, and in histology assessments, some granuloma characteristics can suggest nonspecific dermatitis. Extensive information about the molecular structure and function of leprosy bacillus is now available (Refs 11,12,13), and this has helped in developing molecular techniques for early diagnosis, monitoring of treatment and detection of drug resistance (Refs 10, 14).

\section{Molecular methods for diagnosis}

Probes targeting stretches of DNA (Ref. 15) and ribosomal (r)RNA or rRNA genes of $M$. leprae (Refs $16,17,18,19)$ have been developed by various investigators. The probes targeting DNA need the presence of at least $10^{4}$ copies of target DNA for a positive result (Ref. 15), whereas rRNA-targeting systems can be 10-100-fold more sensitive (Ref. 19); however, because of the small number of organisms present in specimens from PB cases, these probes serve only a limited purpose. During the past 10 years or so, several polymerase chain reaction (PCR) methods have been developed to amplify different gene stretches of $M$. leprae. These include the genes encoding various $M$. leprae proteins [18 kDa (Ref. 15), $36 \mathrm{kDa}$ (Ref. 20), $65 \mathrm{kDa}$ (Refs 21, 22), leprosy serum reactive (LSR) (Ref. 23)] and rRNA (Refs 24, 25), and repetitive sequences (Ref. 26). These assays have been reported to be sensitive to 1-10 organisms and to be positive in $95-100 \%$ of BL/LL and $50-70 \%$ of TT, BL and I specimens (Refs 10, 14). rRNAtargeting probes have been developed into in situ hybridisation protocols and have been found to be of value in confirmation of diagnosis in cases with nonspecific histological features (Mohan Natrajan, Central JALMA Institute for Leprosy, Agra, India, pers. commun.). Therefore, in situ hybridisation and immunohistological approaches (Ref. 27) provide good diagnostic strategies to enhance the sensitivity and specificity of histological diagnosis. Forty to fifty per cent of cases missed by standard histology can be confirmed by the use of molecular methods. Absence of positivity in the remaining cases could reflect the need to further optimise these methods, and/or the possibility that many cases with nonspecific histological features might not be leprosy. These probes and gene amplification assays can be of immense help for the diagnosis of early atypical PB leprosy and also in mass confirmation of diagnosis for epidemiological and research purposes (Refs 10,14).

\section{Molecular methods for monitoring treatment}

As M. leprae has not been cultivated in any acceptable in vitro medium system, time- 
consuming and relatively insensitive animal models have to be used to assess $M$. leprae viability (Ref. 10). Molecular biology has provided an alternative effective technology route for this purpose.

When the PCR technology for detecting $M$. leprae gene sequences was introduced, it was reported that this might be useful both for diagnosis and for assessment of viable load, as a reduction in signals was found to correlate with loss of viability (Ref. 26). These trends were confirmed in subsequent studies (Ref. 28). However, because of the persistence of weak signals in some cases a long time after effective treatment (Refs 10, 29), DNA-based PCR assays appear to have limited application in monitoring treatment, particularly in distinguishing late reactions and relapses.

During recent years, molecular techniques for viability estimation of $M$. leprae have been developed that are based on a quantitative estimation of RNA levels by direct hybridisation with specific probes (Refs 10, 14, 30), or by amplification by PCR (Ref. 31) or isothermal reactions (Ref. 32). The method of grading the positivity levels of $M$. leprae-specific rRNA in the tissues has been useful for monitoring therapeutic responses (Ref. 30). Techniques such as reverse transcription (RT)-PCR targeting 16S rRNA (Refs 10, 31), and nucleic acid sequence-based amplification (NASBA) targeting 16S rRNA (Ref. 32), have been reported to be useful for the determination of viability of $M$. leprae. Cases with positive results indicate the presence of viable organisms and should be considered for antileprosy chemotherapy. Such approaches could also be helpful in differentiating conditions such as late reactions and relapses for patient care.

\section{Molecular methods for monitoring drug resistance}

The lack of a suitable in vitro cultivation system for M. leprae has also hindered assessment of drug resistance. Molecular biology has provided important tools to investigate the molecular mechanisms of drug susceptibility and resistance in leprosy. The use of dapsone and rifampicin as monotherapies to treat leprosy in the 1970s resulted in the rapid emergence of drug resistance. With the introduction of MDT the trend has been apparently reversed and at present drug resistance is not considered a major problem. However, as active surveillance studies have not been carried out, the exact magnitude of drug resistance currently is not known. As in $M$. tuberculosis, mutations in the $M$. leprae rpoB locus are associated with rifampicin resistance (Refs 33, 34). The basis of dapsone resistance appears complex, but mutations in the $M$. leprae folPI locus have been found to be associated with a high degree of dapsone resistance (Ref. 35). PCR is used to directly amplify the target loci $(r p o \mathrm{~B}$ for rifampicin and folPI for dapsone) and mutations are confirmed by techniques such as hybridisation with appropriate probes or sequencing. Little is known about the basis of resistance for drugs such as clofazimine. With the use of new techniques for the detection of mutations directly from clinical specimens, surveillance programmes to determine the exact magnitude of drug-resistant mutants to rifampicin, and possibly other drugs, can be undertaken from the biopsies.

Sequencing of the genome of $M$. leprae has been completed (Ref. 36). The information generated opens new opportunities in functional genomics and proteomics. Such studies will undoubtedly provide scope to develop improved molecular methods for confirmation of diagnosis, for assessing prognosis and for detection of drug resistance.

\section{Current therapy for leprosy}

There have been rapid changes in the treatment of leprosy in the past few decades. The therapeutic scenario has moved from dapsone monotherapy in the 1970s to MDT using drugs such as dapsone, rifampicin and clofazimine. MDT was expected to shorten the length of treatment, leading to better patient compliance, and reduce the problem of drug resistance because of the combined use of multiple drugs with differing modes of action. MDT has indeed revolutionised the treatment of the disease and has been greatly welcomed by patients and doctors alike. More recently, several newer, more-potent drugs and immunomodulators have been introduced in the treatment of leprosy. This has increased the scope for further improvement in the treatment of the disease.

\section{Evolution and current status of WHO MDT regimens}

The World Health Organization (WHO) introduced MDT in 1982, and advocated shortcourse treatment regimens (Ref. 37). According to WHO guidelines, PB patients were to be treated 
with two drugs [rifampicin (600 $\mathrm{mg}$, once a month, supervised) and dapsone (100 mg, daily, unsupervised, for 6 months)]. As most of these patients are lepromin-positive, it was thought that any residual organisms remaining after stoppage of therapy would be taken care of by the immunity of the host. Treatment for MB patients comprised three drugs [rifampicin (600 $\mathrm{mg}$, once a month, supervised), clofazimine (300 $\mathrm{mg}$, once a month, supervised; along with $50 \mathrm{mg}$, daily, unsupervised) and dapsone (100 mg, daily, unsupervised)]. The treatment was to be given for 2 years or until the attainment of smear negativity - whichever was earlier.

In the early 1990s, the concept of fixedduration treatment (FDT) was introduced for control programmes. It was advocated that treatment in PB cases should be stopped after completion of six supervised doses taken in a maximum of 9 months, and treatment in MB cases be stopped after completion of 24 supervised doses in 36 months, irrespective of whether the smears were positive or negative (Ref. 38). This duration has been further reduced to 12 months for $\mathrm{MB}$ cases, and a single-dose regimen comprising rifampicin (600 mg), ofloxacin (400 $\mathrm{mg}$ ) and minocycline (100mg) (ROM) has been recommended for mono-lesion cases (Refs 39, 40). These regimens have already been implemented by control programmes in some countries, such as India (Ref. 41).

Although ROM and 12-month FDT regimens have been introduced only recently, considerable experience has accumulated on the application of various earlier recommended MDT regimens. The overall response has been good. With MDT, there is rapid killing of $M$. leprae and also faster negativity has been observed from the main portal of exit and dissemination - the nose. Confidence in the results has led to the declaration of 'cured' patients, and thus the prevalence of recorded leprosy cases has declined significantly worldwide. Problems of drug resistance using these regimens also appear to be under control. However, some limitations have been consistently observed, and in order to achieve more-effective patient care and control of the disease it is important to discuss these.

\section{Limitations in PB leprosy therapy}

On the whole, treatment in this group of patients appears satisfactory (Ref. 42). There are four issues that require debate, as follows.
First, residual disease activity in the lesions at the end of six months of treatment has been observed in the skin lesions in $10-67 \%$ of patients by various workers (Refs 42, 43, 44, 45, 46, 47). This persisting activity is lower in fresh leprosy cases reporting for treatment recently (Ref. 48). Follow-up studies have shown that this persisting activity does subside in some patients, but in others this worsens and requires further therapy (Refs 43, 44).

Second, there is the issue of late reactions. Late reactions usually occur after 6-18 months, but have also been reported up to 5 years after stoppage of therapy. After discontinuation of therapy, $5-21 \%$ of patients have been reported to suffer from late reactions (Refs $43,45,49$ ). It needs to be emphasised that it is very difficult to distinguish reactions from relapses (Ref. 46). These conditions overlap not only clinically but also histologically. Using the mouse foot-pad assay (Ref. 50) and molecular probes targeting rRNA (Ref. 10), evidence for the presence of live organisms in specimens from cases clinically diagnosed as 'late reaction' has been reported. It is therefore at present too risky to treat these patients with steroids alone for long periods after stoppage of the MDT; additional MDT along with steroids would be more appropriate.

Third is the issue of relapse. The relapses can be defined as the gradual appearance of new lesions with increased reactivity in some or all old lesions, with or without nerve involvement or skin smear positivity. Relapse rates have varied from $0.1-1 \%$ in some studies (Ref. 47) to 6-13\% in others (Refs 43, 44, 49). Some of this variation could be due to the different definition of relapses being used in different studies, and to the difficulty in the diagnosis because of the overlap between relapses and reactions. It has been observed that all the ongoing problems of persisting activity and reactions / relapses can be significantly reduced by extension of MDT (Ref. 45), additional dapsone therapy (Refs 43,49), addition of clofazimine to the drug regimen (Ref. 48) and use of drugs such as prothionamide (Ref. 51). Of these, clofazimine appears to be the most attractive option, as the same regimen, with a different duration, can be administered to $\mathrm{PB}$ and $\mathrm{MB}$ patients. Experience with the use of other drugs such as minocycline in PB leprosy is very limited (Ref. 52). At present, these will perhaps be considered in cases with hypersensitivity and intolerability to conventional drugs. 
Fourth, it is unclear how mono-lesion cases should be treated. After the widespread use of MDT, there has been a change in the profile of the disease, and a substantial proportion of fresh cases are presenting with single lesions as they are being diagnosed early. Such lesions are considered innocuous and capable of self healing. The 7th WHO Expert Committee (Ref. 39) has recommended a single dose of ROM for the treatment of these cases. These recommendations have been accepted by the national programmes in countries such as India (Ref. 41). In a study reported from India, nearly half of the cases treated with ROM and standard treatment with 6 months of PB MDT were inactive after 18 months of follow-up (Ref. 53). Promising results of ROM treatment in cases with two lesions have been subsequently reported (Ref. 54). These are at variance with earlier studies of responses in single-lesion cases from India (Ref. 55), and Malawi (Ref. 46). Such variance in observations indicates that it might be premature to conclude ROM is efficacious for single-lesion cases, which are likely to be heterogeneous and evolving cases. Published follow-up of these cases is inadequate (Ref. 56). Furthermore, there are several theoretical risks from the use of a single-dose treatment (Ref. 57). For example, some bacteria might not be multiplying at the time of administration of the drug and will therefore not be targeted; also, some cases will be $\mathrm{MB}$ or progressing towards $\mathrm{MB}$ disease, for which this single-dose regimen is absolutely inadequate.

\section{Limitations in MB leprosy therapy}

MB patients have a higher bacterial load and, to prevent the emergence of drug-resistant strains, treatment for 2 years or until smear negativity (Ref. 37) was recommended with at least three drugs: rifampicin, clofazimine and dapsone. This regimen has been found to be highly bactericidal and well tolerated, and is widely accepted. With this regimen, the incidence and severity of reactions decreased and the compliance of the patients has improved. Most of the MB patients become smear negative with 24 doses, although the highly bacillated cases require 5-6 years to become smear negative. Various modifications to the WHO regimen have been proposed. No additional benefits of an initial intensive course of rifampicin or monthly loading dose of clofazimine have been observed (Ref. 58). The response to the operationally easier FDT of 24 doses in 36 months has been good (Ref. 38). This regimen has been used worldwide during the past 5-6 years and overall relapses of less than $1 \%$ have been reported (Ref. 59).

Three important issues have emerged from these trials. First, even after 2 years of therapy, viable persisters have been reported in $9-16 \%$ of the initially highly bacillated (BI 3-6+) patients (Refs 60, 61). Relapse rates of $2.9 \%$ after a followup of 3-5 years, increasing to $20 \%$ after a followup of 7.5 years, have been recorded (Ref. 62). Such high relapse rates have also been reported by others (Ref. 63). Second, an important problem in FDT-treated cases is the occurrence of repeated reactions and therefore continuing morbidity after stoppage of treatment. These patients require treatment with steroids, which is given without the cover of MDT. Chemotherapy along with steroids is desirable as a section of these cases are known to harbour live bacilli. Third, on the basis of theoretical considerations and limited published work (Refs 40,64), the WHO and some programmes have recommended stoppage of treatment in $\mathrm{MB}$ patients after 12 doses (over 1 year) using the standard WHO regimen. It has been reported that rates of decline in BI in leprosy in cases treated for 12 months or 24 months were similar (Ref. 64). However, in another study, high relapse rates in patients with high BI defaulting after 12-16 months of therapy have been reported (Ref. 65). Considering the problems of persisters / relapses even with the 24-month regimen (Refs $62,63)$, caution is required with the 12-month duration regimen. Intensive surveillance at least in selected areas for a period of $8-10$ years is required for detection of relapses.

\section{Treatment of MB leprosy with newer or alternative drugs}

Several newer drugs active against $M$. leprae have emerged that are being evaluated to improve the treatment and reduce the duration of treatment in MB leprosy. Prominent among these are: quinolones (pefloxacin, ofloxacin and sparfloxacin, moxifloxacin); ansamycins (rifabutin, KRM-1648); macrolides (clarithromycin); tetracyclines (minocycline), fuscidic acid and other sulphones (brodimoprim). Of these, quinolones, minocyline and clarithromycin appear to be the front runners in providing alternative drug treatment for MB leprosy (Refs $66,67)$. However, experience of clinical application and the establishment of appropriate regimens of 
these newer drugs are limited. Trials have been conducted in MB patients with an intensive shortcourse regimen consisting of daily treatment with $600 \mathrm{mg}$ rifampicin plus $400 \mathrm{mg}$ of ofloxacin for 1 month. The treatment was then stopped and patients followed-up on placebo (Refs 64,68 ). The initial results suggest that the regimen is well tolerated, but high relapse rates have been observed (Refs 68, 69). Trials have also been conducted using the addition of supervised monthly doses of $100 \mathrm{mg}$ minocycline plus 400 mg ofloxacin to the standard MB MDT regimen, with the treatment stopped after 1 year (Ref. 70). The response to the therapy was satisfactory during the treatment and early follow-up period (Ref. 70); however, conclusions can be drawn only after a longer and adequate follow-up.

\section{Role of immunotherapy in the treatment of leprosy}

Besides the presence of a small population of viable organisms ('persisters') after therapy, the problem of persistence of a large pool of dead bacilli is often encountered. Immunomodulators that can stimulate CMI have been applied to reduce this pool. These agents can be divided into three broad groups: drugs, antigenically related mycobacteria, and other immunomodulators (Ref. 71).

In the drug category, levamisole (Ref. 72) and zinc (Ref. 73), when used as an adjunct to dapsone therapy, have been reported to be useful, as seen by clinical improvement in the lesions and a decrease in the incidence and severity of reactions. Although both are considered to be immunopotentiators of CMI, their exact mechanisms of action are not completely understood. Furthermore, these compounds have not been adequately investigated along with MDT.

Antigens of various mycobacteria have been observed to cross-sensitise the immune response to $M$. leprae and this might help in augmenting $\mathrm{CMI}$ in leprosy (Ref. 71). Prominent among these are Bacille-Calmette Guerin (BCG) (Ref. 74), BCG plus killed M. leprae (Ref. 75), Mycobacterium w (Mw) (Refs 74, 76, 77, 78, 79, 80), and Indian Cancer Research Centre (ICRC) bacillus (Ref. 81). BCG, when administered to patients once in 6 monthly repeated injections along with MDT, resulted in a faster killing of bacilli, a more rapid fall in BI, a reduced incidence of reactions and a faster attainment of smear negativity as compared with the control group, who received placebo with the same MDT (Ref. 74). The combination of BCG plus killed M. leprae has been reported to have an immunomodulatory role in I and LL patients, and in lepromin-negative contacts of leprosy patients (Ref. 75). Mw, a cultivable mycobacteria that has antigenic similarities with $M$. leprae, has been investigated in different trials in humans and has been found to be safe and well tolerated. Compared with MDT, MDT plus Mw has been observed to enhance bacterial killing (Ref. 74), clearance of bacilli (Refs $74,76,80)$ and clearance of granuloma (Refs $78,79,80$ ). As the granuloma and bacilli are cleared faster, a reduced severity and frequency of reactions has been observed (Ref. 77). Combined chemotherapy and immunotherapy with ICRC has been shown to significantly accelerate bacterial clearance (Ref. 81). M. vaccae also shares some antigens with $M$. leprae and has been proposed as an immunotherapeutic agent (Ref. 82).

Several other mediators of immune responses, such as transfer factor (Ref. 83) and various cytokines such as recombinant interferon $\gamma$ (IFN- $\gamma$ ) (Refs 84, 85, 86, 87) and interleukin 2 (IL-2) (Ref. 88) have been used to treat leprosy. Transfer factor induced transient effects such as lepromin conversion (from negative to positive), granuloma formation and increased influx of lymphocytes locally (Ref. 83). Intralesional administration of IFN- $\gamma$ in leprosy patients induced accumulation of lymphocytes and monocytes at the local site of injection (Refs 84, $85,86)$. There was a distinct fall in the BI at the local site, with formation of epithelioid granuloma and occurrence of a reversal reaction in some cases, and enhanced bacterial clearance with IFN- $\gamma$ has also been reported. However, repeated doses of IFN- $\gamma$ have to be given intralesionally to induce systemic effects and have been associated with ENL reactions in LL patients (Ref. 87). The administration of IL-2 also accelerated bacterial clearance (Ref. 88); however, these effects were seen only at the local site.

\section{Therapy of reactions}

Efficient management of reactions to prevent nerve damage requires good clinical judgment in making an appropriate early diagnosis and assessing the extent of severity in terms of nerve deficit and multi-organ involvement. The treatment of reactions is based on suppression of inflammation and its consequences. The 
frequency and severity of ENL reactions in BL/ LL cases have been greatly reduced by administration of clofazimine. Many drugs, such as salicylates, non-steroidal anti-inflammatory drugs (NSAIDs), chloroquin, antimonials, steroids, pentoxyphylline, thalidomide and others, are used in the management of ENL (Refs 89,90 ). Steroids continue to provide the mainstay of management of type I reactions in borderline and tuberculoid patients. Standard steroid regimens have been described to control reactions (Ref. 89); however, the standard WHO regimen is considered too short (Ref. 90). The efficacy of drugs such as cyclosporin has been reported to vary from modest to highly effective for the treatment of type I reactions (Ref. 90). The application of molecular tools for quantitative estimation of cytokines by mRNA-based methods and quantitative PCR (Refs 91, 92), is leading to a better understanding of mechanisms and also of the effects of treatment. Earlier compartmentalised concepts of type 1 and type 2 immunity in tuberculoid versus lepromatous cases, respectively, and immune complexes as solely responsible for ENL and DTH for reversal reactions, are now changing with the new evidence. These studies have shown upregulation of T helper 1 (Th1) responses in ENL (Refs 91, 92). A subgroup of patients showing a slower response to steroids that correlates with cytokine profiles (Ref. 92) needs to be followed up to identify patient groups and markers that might help in deciding upon appropriate treatment. Steroid dependence is a serious problem and thalidomide analogues might provide a possible option, although these are still under development (Ref. 93).

\section{Future challenges}

The treatment of leprosy has improved significantly over recent years and this has helped to tackle the disease at the public health level. However, optimal regimens are still evolving. Some of the recently recommended regimens such as single-dose ROM and 12-month FDT need to be kept under close scrutiny for some time, and various modified regimens that have shown promising results need to be considered for improving the therapy. The idea of developing a common regimen for $\mathrm{PB}$ and $\mathrm{MB}$ leprosy is also gaining momentum (Refs $42,48,67$ ) and needs to be pursued. The addition of newer effective drugs such as ofloxacin and minocycline to treatment regimens is increasing, and their potential in effectively reducing the duration of treatment and the management of special situations such as resistance or intolerance is apparent. Many patients require individual attention and tailormade treatment. Indications for such improvisations could be a poor response to standard treatment and hypersensitivity to some of the drugs. For such patients, replacement of the drug(s) might be required (Refs 94, 95). Currently, several WHO- and ILEP-sponsored trials to monitor various regimens, including some new alternatives, are progressing (Ref. 95).

As the total patient load has been considerably reduced, easy diagnositc methods such as skin smears for acid-fast bacilli should be reintroduced for monitoring of cases at field-level clinics. Molecular methods should be available at reference laboratories and be more extensively used in research and epidemiological studies.

Leprosy has been a feared disease mainly because of the deformities associated with it. After widespread use of MDT, there has been a sea change in the profile of the disease. Early and appropriate treatment undoubtedly helps in reducing the severity and frequency of deformities. Nevertheless, disabilities continue to be a major problem (Ref. 96). Different preventive (management of reactions, nerve decompression) and corrective (tendon transfers, management of plantar ulcers) procedures to manage deformities are available. Besides the availability of surgery, timely physiotherapy and health education are very important in the prevention, management and rehabilitation resulting from the disabilities. As leprosy patients continue to have disabilities for a long time or even life, these services will be required for a much longer time.

Genuine concerns have been raised about the continued high-incidence rates of leprosy even in areas with intensive MDT campaigns for 10-15 years. The issues of inadequate coverage, nonhuman sources, extraordinarily long incubation periods and effectiveness of regimens being used need to be analysed for these unexpected results. The rich experience of the past of treating leprosy patients with different regimens, together with lessons from careful follow-up of new regimens and the appropriate use of molecular tools for early diagnosis and surveillance of drug resistance, provide an excellent base from which to progress towards the goals of sensitive and specific diagnostics as well as optimal regimens 
for all leprosy patients. The knowledge emanating from analysis of the human genome and the M. leprae and M. tuberculosis genomes (Ref. 97) will undoubtedly strengthen the development of relevant technologies for more-effective management of leprosy at patient and public health levels.

\section{Acknowledgements and funding}

I thank the various scientific and technical colleagues who participated in the different studies cited in this article. I also gratefully acknowledge the financial support of the Indian Council of Medical Research, the Department of Science and Technology, the Department of Biotechnology, the World Health Organization (WHO) and LEPRA. My thanks to Prof. Ben Naafs (Gracht 15, 8485 KN Munnekeburen, The Netherlands) and Prof. Bhushan Kumar (PGI, Chandigarh, India) for peer review of this manuscript, and Sh. S. K. Kulshrestha, for secretarial assistance in its preparation.

\section{References}

1 Shepard, C.C. (1960) The experimental disease that follows the injection of human leprosy bacilli into foot pads of mice. J Exp Med 112, 445-454

2 Cree, I.A. and Smith, W.C. (1998) Leprosy transmission and mucosal immunity: towards eradication? Lepr Rev 69, 112-121, PubMed ID: 98381384

3 Pfaltzgraff, R.E. and Ramu, G. (1994) Clinical leprosy. In Leprosy (Hastings, R.C., ed.), pp. 237290, Churchill Livingstone, London

4 Kumar, B., Rai, R. and Kaur, I. (2000) Systemic involvement in leprosy and its significance. Indian J Lepr 72, 123-142, PubMed ID: 20390875

5 Dharmendra (1994) Classifications of leprosy. In Leprosy (Hastings, R.C., ed.), pp. 179-190, Churchill Livingstone, London

6 Chacko, C.J. (2000) Leprosy: pathology. In IADVL Textbook and Atlas of Dermatology (Valia, R.G. and Valia, A.R., eds), pp. 1563-1572, Bhalani Publishing House, Mumbai, India

7 Rees, R.J.W. and Young, D.B. (1994) Microbiology of leprosy. In Leprosy (Hastings, R.C., ed.), pp. 49-86, Churchill Livingstone, London

8 Ridley, D.S. (1964) Bacterial indices. In Leprosy in Theory and Practice (Cochrane, R.G. and Davey, T.F., eds), pp. 620-622, John Wright and Sons, Bristol

9 WHO Expert Committee on Leprosy (1988) 6th Report. Tech Rep Ser 768, World Health
Organization

10 Katoch, V.M. (1998) New investigative techniques in leprosy. In Dermatology Update (Valia, R.G. and Valia, A.R., eds), pp. 165-174, Bhalani Publishing House, Mumbai, India

11 Clark-Curtiss, J.E. et al. (1985) Molecular analysis of DNA and construction of genomic libraries of Mycobacterium leprae. J Bacteriol 161, 1093-1102

12 Young, R.A. et al. (1985) Genes for the major protein antigens of the leprosy parasite Mycobacterium leprae. Nature 316, 450-452, PubMed ID: 85268051

13 Clark-Curtiss, J.E. (1990) Genome structure of mycobacteria. In Molecular Biology of Mycobacteria (McFadden, J., ed.), pp. 77-98, Surrey University Press, Surrey

14 Katoch, V.M. (1999) Molecular techniques for leprosy: present applications and future perspective. Indian J Lepr 71, 45-60

15 Williams, D.L. et al. (1990) The use of a specific DNA probe and polymerase chain reaction for the detection of Mycobacterium leprae. J Infect Dis 162, 193-200, PubMed ID: 90285512

16 Katoch, V.M. et al. (1994) Progress in developing ribosomal RNA and rRNA gene (s) based probes for the diagnosis and epidemiology of infectious diseases including leprosy. In Tropical Diseases Molecular Biology and Control Strategies

(Kumar, S., ed.), pp. 581-587, Council of Scientific and Industrial Research, New Delhi, India

17 Pitulle, C., Stackebrandt, E. and Kazda, J. (1990) Further evidence for the exclusiveness of the Mycobacterium leprae-specific DNA probe. Int J Lepr Other Mycobact Dis 58, 130-133, PubMed ID: 90203771

18 Teske, A., Wolters, J. and Bottger, E.C. (1991) The $16 \mathrm{~S}$ rRNA nucleotide sequence of Mycobacterium leprae: phylogenetic position and development of DNA probes. FEMS Microbiol Lett 64, 231-237, PubMed ID: 91357438

19 Sharma, R.K. et al. (1996) Comparison of probes targeting ribosomal RNA vs DNA in leprosy cases. Indian J Med Microbiol 14, 99-104

20 Hartskeerl, R.A., de Wit, M.Y. and Klatser, P.R. (1989) Polymerase chain reaction for the detection of Mycobacterium leprae. J Gen Microbiol 135, 2357-2364, PubMed ID: 90188277

21 Plikaytis, B.B., Gelber, R.H. and Shinnick, T.M. (1990) Rapid and sensitive detection of Mycobacterium leprae using a nested- primer gene amplification assay. J Clin Microbiol 28, 1913-1917, PubMed ID: 91035969

22 Hackel, C. et al. (1990) Specific identification of 
Mycobacterium leprae by polymerase chain reaction. Mol Cell Probes 4, 205-210

23 Misra, N. et al. (1995) Clinical utility of LSR/ A15 gene for Mycobacterium leprae detection in leprosy tissues using the polymerase chain reaction. Int J Lepr Other Mycobact Dis 63, 35-41, PubMed ID: 95248198

24 Cox, R.A. et al. (1991) The $16 \mathrm{~S}$ ribosomal RNA of Mycobacterium leprae contains a unique sequence which can be used for identification by the polymerase chain reaction. J Med Microbiol 35, 284-290, PubMed ID: 92045980

25 Pattyn, S.R. et al. (1992) Polymerase chain reaction amplifying DNA coding for speciesspecific rRNA of Mycobacterium leprae. Int J Lepr Other Mycobact Dis 60, 234-243, PubMed ID: 92395349

26 Woods, S.A. and Cole, S.T. (1989) A rapid method for the detection of potentially viable Mycobacterium leprae in human biopsies: a novel application of PCR. FEMS Microbiol Lett 53, 305-309, PubMed ID: 90128210

27 Natrajan, M., Katoch, K. and Katoch, V.M. (1999) Histology and immuno-histology of lesions clinically suspicious of leprosy. Acta Leprol 11, 93-98, PubMed ID: 20011964

28 Kampirapap, K. et al. (1998) DNA amplification for detection of leprosy and assessment of efficacy of leprosy chemotherapy. Int J Lepr Other Mycobact Dis 66, 16-21, PubMed ID: 98277040

29 Singh, H.B. et al. (1999) Effect of treatment on PCR positivity in multibacillary leprosy patients treated with conventional and newer drugs ofloxacin and minocycline. Acta Leprol 11, 179182, PubMed ID: 20443118

30 Sharma, R.K. et al. (1997) Microdensitometric scanning procedure for quantitative assessment of hybridization of rRNA targeting probes in leprosy. Acta Leprol 10, 213-217, PubMed ID: 98108515

31 Kurabachew, M., Wondimu, A. and Ryon, J.J. (1998) Reverse transcription-PCR detection of Mycobacterium leprae in clinical specimens. J Clin Microbiol 36, 1352-1356, PubMed ID: 98233891

32 van der Vliet, G.M. et al. (1996) Use of NASBA RNA amplification for detection of Mycobacterium leprae in skin biopsies from untreated and treated leprosy patients. Int J Lepr Other Mycobact Dis 64, 396-403, PubMed ID: 97182058

33 Honore, N. and Cole, S.T. (1993) Molecular basis of rifampin resistance in Mycobacterium leprae. Antimicrob Agents Chemother 37, 414-418, PubMed ID: 93213109

34 Williams, D.L. and Gillis, T.P. (1999) Detection of drug-resistant Mycobacterium leprae using molecular methods. Indian J Lepr 71, 137-153, PubMed ID: 99368189

35 Gillis, T.P. and Williams, D.L. (2000) Dapsone resistance in Mycobacterium leprae. Lepr Rev 71 Suppl, S91-95, PubMed ID: 21072040

36 Cole, S.T. et al. (2001) Massive gene decay in the leprosy bacillus. Nature 409, 1007-1011, PubMed ID: 21128732

37 WHO Study Group (1982) Chemotherapy of Leprosy for Control Programmes. Tech Rep Ser 675, World Health Organization

38 WHO Study Group (1994) Chemotherapy of Leprosy. Tech Rep Ser 847, World Health Organization

39 WHO Expert Committee on Leprosy (1997) Recommendations of the 7th WHO Expert Committee on the Chemotherapy of Leprosy. Tech Rep Ser 874, World Health Organization

40 Ji, B. (1998) Why multidrug therapy for multibacillary leprosy can be shortened to 12 months. Lepr Rev 69, 106-109, PubMed ID: 98381382

41 Directorate General (Leprosy Division), Nirman Bhawan (1997) Modified guidelines on MDT regimen to be followed under NLEP. L. 19025/6/ 97, Government of India, New Dehli

42 Katoch, K. (2000) Therapeutic prospects for paucibacillary leprosy. Indian J Lepr 72, 351-361, PubMed ID: 20556904

43 Katoch, K. et al. (1989) Relapses in paucibacillary patients after treatment with three short- term regimens containing rifampin. Int J Lepr Other Mycobact Dis 57, 458-464, PubMed ID: 89310087

44 Pavithran, K. (1988) Relapse of paucibacillary leprosy after short course multidrug therapy. Indian J Lepr 60, 225-229, PubMed ID: 89054894

45 Bhate, R.D. et al. (1986) Experience with multidrug therapy in paucibacillary leprosy. Indian J Lepr 58, 244-250, PubMed ID: 87110928

46 Boerrigter, G, Ponnighaus, J.M. and Fine, P.E. (1988) Preliminary appraisal of a WHOrecommended multiple drug regimen in paucibacillary leprosy patients in Malawi. Int J Lepr Other Mycobact Dis 56, 408-417, PubMed ID: 88332074

47 Becx-Bleumink, M. (1992) Relapses in leprosy patients after release from dapsone monotherapy; experience in the leprosy control 
program of the all Africa Leprosy and

Rehabilitation Training Center (ALERT) in

Ethiopia. Int J Lepr Other Mycobact Dis 60, 161-

172, PubMed ID: 92395341

48 Katoch, K. et al. (1999) Chemotherapy trial in paucibacillary leprosy using clofazimine. Indian J Lepr 71, 311-324, PubMed ID: 20091682

49 Grugni, A. et al. (1990) Relapses in paucibacillary leprosy after MDT - a clinical study. Int J Lepr Other Mycobact Dis 58, 19-24, PubMed ID: 90203773

50 Shetty, V.P., Wakade, A. and Antia, N.H. (2001) A high incidence of viable Mycobacterium leprae in post-MDT recurrent lesions in tuberculoid leprosy patients. Lepr Rev 72, 337-344, PubMed ID: 21572708

51 Katoch, K. et al. (1992) Treatment of paucibacillary leprosy with a regimen containing rifampicin, dapsone and prothionamide. Indian J Lepr 64, 303-312, PubMed ID: 93056787

52 Pavithran, K. (1992) Minocycline cures tuberculoid leprosy. Lepr Rev 63, 291-292, PubMed ID: 93023526

53 (1997) Efficacy of single dose multidrug therapy for the treatment of single- lesion paucibacillary leprosy. Single-lesion Multicentre Trial Group. Indian J Lepr 69, 121-129, PubMed ID: 97436296

54 (2001) A comparative trial of single dose chemotherapy in paucibacillary leprosy patients with two to three skin lesions. Indian J Lepr 73, 131-143, PubMed ID: 21464104

55 Katoch, K. et al. (1995) Response of leprosy patients with single lesions to MDT. Acta Leprol 9, 133-137, PubMed ID: 95358099

56 Gupte, M.D. (2000) Field trials of a single dose of the combination rifampicin-ofloxacinminocycline (ROM) for the treatment of paucibacillary leprosy. Lepr Rev 71 Suppl, S7780, PubMed ID: 21072037

57 Katoch, V.M. (1998) Is there a microbiological rationale for single-dose treatment of leprosy? Lepr Rev 69, 2-5, PubMed ID: 98291561

58 Katoch, K. et al. (1989) Results of a modified WHO regimen in highly bacilliferous BL/LL patients. Int J Lepr Other Mycobact Dis 57, 451457, PubMed ID: 89310086

59 WHO Leprosy Unit (1994) Risk of Relapse in Leprosy. WHO/CTD/Lep/94.1, World Health Organization

60 Subcommittee on clinical trials of scientific working group on chemotherapy of leprosy (THELEP) of UNDP/World Bank/WHO special programme for research in tropical diseases
(1987) THELEP controlled clinical drug trials. Int J Lepr 55 (Suppl. 4), 864-868

61 Katoch, V.M. et al. (1989) Effect of chemotherapy on viability of Mycobacterium leprae as determined by ATP content, morphological index and FDA-EB fluorescent staining. Int J Lepr Other Mycobact Dis 57, 615-621, PubMed ID: 89381528

62 Jamet, P. and Ji, B. (1995) Relapse after long-term follow up of multibacillary patients treated by WHO multidrug regimen. Marchoux Chemotherapy Study Group. Int J Lepr Other Mycobact Dis 63, 195-201, PubMed ID: 95325736

63 Girdhar, B.K., Girdhar, A. and Kumar, A. (2000) Relapses in multibacillary leprosy patients: effect of length of therapy. Lepr Rev 71, 144-153, PubMed ID: 20375998

64 Ganapati, R. et al. (1997) Rate of decline in bacterial index in leprosy; observations after three different chemotherapeutic interventions. Int J Lepr Other Mycobact Dis 65, 264-266, PubMed ID: 97395466

65 Katoch, K. et al. (1991) Clinical and bacteriological progress of highly bacillated BLLL patients discontinuing treatment after different periods of MDT. Int J Lepr Other Mycobact Dis 59, 248-254, PubMed ID: 91302952

66 Ji, B. and Grosset, J.H. (1990) Recent advances in the chemotherapy of leprosy. Lepr Rev 61, 313329, PubMed ID: 91125049

67 Grosset, J. (2000) The new challenges for chemotherapy research. Lepr Rev 71 Suppl, S100104, PubMed ID: 21072042

68 Ganapati, R. et al. (1998) Relapse of multibacillary leprosy after rifampin and ofloxacin treatment for 28 days; a case report. Int J Lepr Other Mycobact Dis 66, 56-58, PubMed ID: 98277047

69 Ji, B. et al. (1997) High relapse rate among lepromatous leprosy patients treated with rifampin plus ofloxacin daily for 4 weeks. Antimicrob Agents Chemother 41, 1953-1956, PubMed ID: 97447652

70 Katoch, K. et al. (2000) Chemotherapy trials in MB leprosy using conventional and newer drugs ofloxacin and minocycline. Indian J Dermatol Venerol Leprol 66, 18-25

71 Katoch, K. (1996) Immunotherapy of leprosy. Indian J Lepr 68, 349-361, PubMed ID: 97155189

72 Ramu, G. and Sengupta, U. (1983) Preliminary trial of intervention levamisole therapy in persistently bacteriologically positive lepromatous leprosy. Lepr India 55, 64-67, 
PubMed ID: 83269815

73 Mathur, N.K. et al. (1984) Oral zinc as an adjunct to dapsone in lepromatous leprosy. Int J Lepr Other Mycobact Dis 52, 331-338, PubMed ID: 85005925

74 Katoch, K. et al. (1995) Treatment of bacilliferous BL/LL cases with combined chemotherapy and immunotherapy. Int J Lepr Other Mycobact Dis 63, 202-212, PubMed ID: 95325737

75 Convit, J. et al. (1982) Immunotherapy with a mixture of Mycobacterium leprae and BCG in different forms of leprosy and in Mitsudanegative contacts. Int J Lepr Other Mycobact Dis 50, 415-424, PubMed ID: 83184923

76 Zaheer, S.A. et al. (1993) Combined multidrug and Mycobacterium $w$ vaccine therapy in patients with multibacillary leprosy. J Infect Dis 167, 401-410, PubMed ID: 93132391

77 Kar, H.K. et al. (1993) Reversal reaction in multibacillary leprosy patients following MDT with and without immunotherapy with a candidate for an antileprosy vaccine,

Mycobacterium w. Lepr Rev 64, 219-226,

PubMed ID: 94048880

78 Mukherjee, A. et al. (1992) Histopathological monitoring of an immunotherapeutic trial with Mycobacterium w. Int J Lepr Other Mycobact Dis 60, 28-35, PubMed ID: 92291589

79 Natrajan, M. et al. (1992) Histological changes with combined chemotherapy and immunotherapy in highly bacillated lepromatous leprosy. Acta Leprol 8, 79-86

80 De Sarkar, A. et al. (2001) Impact of combined Mycobacterium $w$ vaccine and 1 year of MDT on multibacillary leprosy patients. Int J Lepr Other Mycobact Dis 69, 187-194, PubMed ID: 21865501

81 Bhatki, W.S. and Chulawala, R.G. (1992) Immunotherapeutic potential of ICRC vaccine: a case control study. Lepr Rev 63, 358-364, PubMed ID: 93125077

82 Stanford, J.L. et al. (1990) Mycobacterium vaccae in immunoprophylaxis and immunotherapy of leprosy and tuberculosis. Vaccine 8, 525-530, PubMed ID: 91205864

83 Hastings, R.C. and Job, C.K. (1978) Reversal reactions in lepromatous leprosy following transfer factor therapy. Am J Trop Med Hyg 27, 995-1004, PubMed ID: 79060064

84 SivaSai, K.S. et al. (1993) Effect of recombinant interferon gamma administration on lesional monocytes / macrophages in lepromatous leprosy patients. Int J Lepr Other Mycobact Dis 61, 259269, PubMed ID: 93381368
85 Kaplan, G. et al. (1989) Effect of multiple interferon gamma injections on the disposal of Mycobacterium leprae. Proc Natl Acad Sci U S A 86, 8073-8077, PubMed ID: 90046740

86 Mathur, N.K. et al. (1992) Long-term follow up of lepromatous leprosy patients receiving intralesional recombinant gamma-interferon. Int J Lepr Other Mycobact Dis 60, 98-100, PubMed ID: 92291605

87 Sampaio, E.P. et al. (1992) Prolonged treatment with recombinant interferon gamma induces erythema nodosum leprosum in lepromatous leprosy patients. J Exp Med 175, 1729-1737, PubMed ID: 92268822

88 Kaplan, G. et al. (1991) The systemic influence of recombinant interleukin 2 on the manifestations of lepromatous leprosy. J Exp Med 173, 993-1006, PubMed ID: 91178460

89 Naafs, B. (1996) Bangkok Workshop on Leprosy Research. Treatment of reactions and nerve damage. Int J Lepr Other Mycobact Dis 64, S2128, PubMed ID: 97182073

90 Naafs, B. (2000) Current views on reactions in leprosy. Indian J Lepr 72, 97-122, PubMed ID: 20390874

91 Nath, I. et al. (2000) Dysregulation of IL-4 expression in lepromatous leprosy patients with and without erythema nodosum leprosum. Lepr Rev 71 Suppl, S130-137, PubMed ID: 21072048

92 Lockwood, D.N. (2000) Steroids in leprosy type 1 (reversal) reactions: mechanisms of action and effectiveness. Lepr Rev 71 Suppl, S111-114, PubMed ID: 21072044

93 Kaplan, G. (2000) Potential of thalidomide and thalidomide analogues as immunomodulatory drugs in leprosy and leprosy reactions. Lepr Rev 71 Suppl, S117-120, PubMed ID: 21072046

94 Jacobson, R.R. (1996) Needed research in chemotherapy of leprosy related to the individual patient. Int J Lepr Other Mycobact Dis 64, S16-20, PubMed ID: 97182072

95 Daumerie, D. (2000) Current World Health Organization-sponsored studies in the chemotherapy of leprosy. Lepr Rev 71 Suppl, S88-90, PubMed ID: 21072039

96 Srinivasan, H. (2000) Disability and rehabilitation in leprosy: issues and challenges. Indian J Lepr 72, 317-337, PubMed ID: 20556902

97 Curtiss, R., 3rd et al. (2001) Leprosy research in the post-genome era. Lepr Rev 72, 8-22, PubMed ID: 21254940

98 WHO (2002) Leprosy. Global situation. Wkly Epidemiol Rec 77, 1-8, PubMed ID: 21667176 


\section{Further reading, resources and contacts}

The WHO's Action Programme for the Elimination of Leprosy provides current information on therapy, endemic countries, research and publications.

http://www.who.int/lep/

LEPRA is a UK-based medical development charity whose prime objective is to eradicate leprosy.

http://www.lepra.org.uk/

The Novartis Foundation for Sustainable Development 'has been actively involved in leprosy programs in Asia, Africa and Latin America in partnership with local health authorities, the WHO and nongovernmental agencies'. The efforts of the foundation focus primarily on eliminating leprosy, and their website is a useful source of information on the diagnosis, treatment and elimination of the disease.

http://www.novartisfoundation.com/leprosy/index.htm

Leproma is a powerful web-based tool for extracting information on gene structure and function from a Mycobacterium leprae genome database, using programmes such as BLAST and FASTA.

http://genolist.pasteur.fr/Leproma

\section{Features associated with this article}

\section{Figure}

Figure 1. Global leprosy situation in 2000 (fig001vka).

Table

Table 1. Leprosy situation in 2000 by WHO regions (tab001vka).

\section{Citation details for this article}

Vishwa M. Katoch (2002) Advances in the diagnosis and treatment of leprosy. Exp. Rev. Mol. Med. 22 July http://www.expertreviews.org/02004763h.htm 